\title{
Anemia ferropriva em atletas adolescentes da Fundação Vila Olímpica de Manaus-AM
}

\author{
Sandra Maria Trindade NUNES ${ }^{1}$, Lucia Kiyoko Ozaki YUYAMA², Dartagnan Pinto GUEDES³, \\ Maria Conceição de OLIVEIRA ${ }^{4}$
}

\section{RESUMO}

Considerando a escassez de informações quanto ao estado nutricional de atletas jovens, identificou-se a prevalência de anemia ferropriva e sua associação com indicadores nutricionais de atletas adolescentes participantes do Programa de Iniciação Esportiva da Fundação Vila Olímpica de Manaus-AM. A amostra foi constituída por 194 atletas adolescentes do gênero masculino, voluntários, praticantes de diferentes modalidades esportivas classificados como iniciantes. A coleta dos dados foi realizada mediante verificação de medidas antropométricas (peso corporal, estatura e espessuras das dobras cutâneas tricipital e subescapular), dosagem de hemoglobina utilizando o hemoglobinômetro portátil (Sistema Hemocue) e instrumento socioeconômico. No diagnóstico do estado nutricional foram utilizados os indicadores Estatura para a Idade (E/I) e Índice de Massa Corporal para a idade (IMC/I), assumindo pontos de corte propostos pela OMS. Os resultados mostraram que 9,4\% dos adolescentes apresentaram desnutrição, 8,2\% sobrepeso e 4,6\% obesidade. A prevalência de anemia ferropriva encontrada na amostra foi de $41,7 \%$, com predominância na classe socioeconômica mais baixa. Embora a prevalência de anemia tenha sido elevada, o estado nutricional dos adolescentes não influenciou sobre este resultado, considerando que a maioria dos adolescentes apresentou-se eutrófica. Diante do exposto, sugere-se a adoção de medidas efetivas de intervenção e de educação nutricional visando à minimização e/ou o controle da anemia ferropriva.

PALAVRAS CHAVE: Anemia ferropriva, Adolescentes, Atletas, Manaus.

\section{Iron deficiency anaemia in adolescent athletes of the Vila Olímpica Fonndation of Manaus - AM}

\begin{abstract}
Considering the dearth of information with regard to the nutritional state of young athletes, were identified the prevalence of iron deficiency anaemia and its association with nutritional indicators of adolescent athletes participating in the Sport Initiation Program of the Vila Olímpica Foundation in Manaus -AM. A sample was made up of 194 voluntary adolescent male athletes classified as beginners, who participated in several different sports. Data collected was by verification of anthropometric measures (body weight, height, and skinfold thicknesses of triceps and subscapular skinfold), hemoglobin dosage utilizing a portable hemoglobinometer, HemoCue direct calorimetric method, and socioeconomic instrument. In the diagnosis of the nutritional states, we used Height-for-age- and Body Mass Index for the age indicators, adjusted cut-off points proposed by the WHO. The results showed that $9.4 \%$ of the adolescents suffered from malnutrition, $8.2 \%$ overweight, and 4.6 obesity. The prevalence of iron deficiency anaemia found in the samples was $41.7 \%$, with predominance in the lowest socioeconomic class. Although the prevalence of anaemia was high, the nutritional state of the adolescents did not influence these results, considering that the majority looked healthy. In view of these findings, we suggest that effective means be taken for nutritional education to minimize and/or control iron deficiency anaemia.
\end{abstract}

KEY-WORDS: Iron deficiency anaemia, Adolescents, Athletes, Manaus.

\footnotetext{
${ }^{1}$ Mestre em Ciências de Alimentos pela Faculdade de Ciências da Saúde/Universidade Federal do Amazonas - UFAM. Rua. Alexandre Amorim 330, Aparecida. Manaus, AM. CEP 69010-300.

2 Instituto Nacional de Pesquisas da Amazônia, Coordenação de Pesquisas em Ciências da Saúde. INPA/CPCS. Av. André Araújo 2936. Aleixo, CEP 690860-001. Manaus, AM. Orientador.

${ }^{3}$ Universidade Estadual de Londrina, Departamento de Fundamentos da Educação Física/UEL. Av. Maringá, 725 sala 304, CEP. 86060-000. Londrina, PR.

${ }^{4}$ Universidade Federal do Amazonas, Departamento de Saúde Coletiva /UFAM. Rua. Alexandre Amorim 330, Aparecida, CEP. 69010-300. Manaus, AM.
} 


\section{INTRODUÇÃO}

As anemias nutricionais constituem um grave problema de saúde pública em todo o mundo e estão associadas ao retardo no desenvolvimento neuropsicomotor, ao comprometimento de imunidade celular e à diminuição da capacidade intelectual (MS, 2007).

A anemia ferropriva é doença de alta prevalência no mundo, atingindo indiscriminadamente tanto o sexo masculino quanto o feminino, em países desenvolvidos ou em desenvolvimento (Oliveira \& Figueiredo, 1998). Estimase que a anemia afete metade dos escolares e adolescentes nos países em desenvolvimento e que suas principais causas sejam as infestações parasitárias e o baixo consumo de ferro (Tsuyoka et al., 1999).

No Brasil, apesar da escassez de dados de base populacional, em revisão de estudos localizados, estima-se uma taxa de $20 \%$ de anemia entre adolescentes (MS, 2004).

Deve-se considerar que, as mudanças da adolescência, como o estirão de crescimento físico e o desenvolvimento sexual e ósseo, promovem o aumento das necessidades de ferro neste estágio de vida (Eisenstein, 2000). A anemia por deficiência de ferro é um diagnóstico freqüente em adolescentes desnutridos, atletas e em esportistas (McArdle et al., 1998).

A ingestão insuficiente de ferro por parte dos atletas pode prejudicar a capacidade de transporte do oxigênio, interferindo no treinamento esportivo e diminuindo, por sua vez, o desempenho atlético (Thompson, 1998).

No Amazonas, estudos envolvendo adolescentes são limitados e os descritos na literatura procuram abordar os não-atletas quanto ao crescimento físico (Silva, 1992), aos hábitos e às preferências alimentares (Doyle \& Feldman, 1997) e à ocorrência de anemia ferropriva (Lima, 2002). No campo esportivo, pouco se sabe sobre o estado nutricional dos atletas Amazonenses.

$\mathrm{Na}$ Fundação Vila Olímpica de Manaus-AM vem sendo desenvolvido o Programa de Iniciação Esportiva com objetivo de incentivar jovens da comunidade a praticarem regularmente esportes para desenvolver e aprimorar suas habilidades e participarem de competições. No entanto, não foram identificados estudos de diagnósticos nutricionais destes jovens quando ingressam no Programa de Iniciação Esportiva. Considerando a escassez de informaçóes quanto ao estado nutricional de atletas amazonenses, o objetivo do presente estudo foi verificar a prevalência de anemia ferropriva, associando-a com indicadores nutricionais de atletas adolescentes participantes do Programa de Iniciação Esportiva da Fundação Vila Olímpica de Manaus-AM.

\section{MATERIAL E MÉTODOS}

A amostra estudada abrangeu 194 atletas adolescentes voluntários do gênero masculino, com idades entre 10 e 18 anos, de diferentes modalidades esportivas e classificados como iniciantes. Essa amostra correspondeu a 50\% do total que estava ingressando no programa. Os adolescentes foram divididos em dois estágios de vida, 10-13 anos e 14-18 anos. Os atletas participavam de sessões com duração de 1 hora de treino, duas ou três vezes por semana.

A coleta dos dados foi realizada mediante verificação de medidas antropométricas, dosagem da hemoglobina e questionário socioeconômico.

Para classificar os atletas adolescentes de acordo com a condição socioeconômica foi utilizada a proposta sugerida pela Associação Brasileira de Institutos de Pesquisa de Mercado (Camargo \& Santos, 1997).

As medidas de peso corporal, estatura e espessuras das dobras cutâneas tricipital e subescapular acompanharam os procedimentos recomendados pelo Anthropometric Standardization Reference (Heyward \& Stolarczyk, 2000).

Para a avaliação do estado nutricional dos participantes foram utilizados indicadores associados à Estatura/Idade (E/I), e Índice de Massa Corporal/Idade (IMC/I). Para tanto, foram utilizados os padróes de referência apresentados pelo National Center for Health Statistics (2000) e pontos de corte preconizados pela World Health Organization (1995).

Para proceder ao diagnóstico associado à anemia foi realizada a dosagem de hemoglobina $(\mathrm{Hb})$ em $100 \%$ da amostra mediante coleta de sangue capilar. A leitura foi viabilizada no ato da coleta utilizando o hemoglobinômetro portátil Sistema Hemocue.

Para verificar a prevalência de anemia foi utilizado o critério diagnóstico proposto pelo consenso do Fundo das Naçōes Unidas para a Infância - UNICEF, United Nations University - UNU, World Health Organization - WHO, Micronutrient Iniciative - MI (1998), que considera os pontos de corte referentes à hemoglobina de acordo com os ciclos de vida: 10 a 11 anos: < 11,5 g. $\mathrm{dl}^{-1}$; $12-13$ anos: $<12,0$ g.dl ${ }^{-1}$; e maior que 13 anos: $<13,0$ g. $\mathrm{dl}^{-1}$.

\section{RESULTADOS E DISCUSSÃO}

A Tabela 1 mostra os valores dos indicadores antropométricas relacionados ao peso corporal, à estatura, ao Índice de Massa Corporal (IMC) e dobras cutâneas dos atletas adolescentes. A classe socioeconômica predominante foi a C $(63,4 \%)$.

Quanto ao estado nutricional os resultados mostraram que a maioria dos atletas adolescentes estava eutróficos $(77,8 \%)$. 
No entanto, a desnutrição foi diagnosticada em 9,4\% dos adolescentes, o sobrepeso em $8,2 \%$ e a obesidade em $4,6 \%$ (Tabela 2).

Os valores relacionados ao percentual de gordura corporal segundo o estágio de vida demonstram que a maioria dos adolescentes $(51,5 \%)$ apresentou um nível esperado de gordura corporal. Porém, uma classificação de baixo nível de gordura esteve presente em 20,6\% dos jovens e de alto nível de gordura em 27,8 \%. Essas diferenças não foram consideradas estatisticamente significantes, porém a proporção de adolescentes que apresentam quantidades de gordura além ou aquém dos limites adequados chama a atenção por serem bastante expressivos, e podem caracterizar desvios nutricionais como desnutrição ou obesidade.

Von Der Heyde (1999), avaliou o estado nutricional de atletas adolescentes por meio do IMC e a eutrofia foi constatada em $86,2 \%$ do gênero masculino, resultado superior ao encontrado no presente estudo. Em um estudo realizado com atletas infanto-juvenis no município de Camboriú, SC, verificou-se um IMC médio de $16,9 \mathrm{~kg} . \mathrm{m}^{-2}$, e predominância de eutrofia em $85 \%$ dos participantes (Nascimento et al., 2004).

Os resultados quanto à concentração de hemoglobina por estágio de vida demonstram uma média de $12,5 \mathrm{~g} \cdot \mathrm{dl}^{-1}$ no estágio de 10-13 anos e 13,3 g.dl-1 ${ }^{-1}$ no estágio de 14-18 anos ( Tabela 3 ).

Tabela 1 - Indicadores antropométricos quanto ao estado nutricional dos atletas adolescentes da Fundação Vila Olímpica de Manaus-AM, 2003.

\begin{tabular}{lllll}
\hline & Média \pm DP & Mediana & Mínimo & Máximo \\
\hline Peso $(\mathrm{kg})$ & $47,7 \pm 14,3$ & 45,8 & 24,4 & 115,5 \\
Estatura $(\mathrm{m})$ & $1,60 \pm 0,1$ & 1,50 & 1,2 & 1,9 \\
IMC $\left(\mathrm{kg} \cdot \mathrm{m}^{-2}\right)$ & $19,2 \pm 3,3$ & 18,5 & 12,3 & 39,1 \\
Tricipital $(\mathrm{mm})$ & $10,0 \pm 5,6$ & 9,0 & 3,0 & 31,0 \\
Subescapular $(\mathrm{mm})$ & $10,2 \pm 4,7$ & 9,0 & 4,0 & 30,0 \\
\hline
\end{tabular}

$\mathrm{DP}=$ Desvio padrão

IMC = Índice de Massa Corporal

Tabela 2 - Anemia ferropriva segundo o estado nutricional dos atletas adolescentes da Fundação Vila Olímpica de Manaus-AM, 2003.

\begin{tabular}{lllllll}
\hline $\begin{array}{l}\text { Estado } \\
\text { Nutricional }\end{array}$ & \multicolumn{2}{l}{ Anêmicos } & \multicolumn{2}{l}{ Não-Anêmicos } & Total & \\
\hline & $\mathrm{N}$ & $\%$ & $\mathrm{n}$ & $\%$ & $\mathrm{n}$ & $\%$ \\
Baixo Peso & 7 & 3,6 & 11 & 5,7 & 18 & 9,4 \\
Eutrofia & 63 & 32,5 & 88 & 45,3 & 151 & 77,8 \\
Risco de & 5 & 2,6 & 11 & 5,7 & 16 & 8,2 \\
sobrepeso & & & & & & \\
Obesidade & 6 & 3,0 & 3 & 1,5 & 09 & 4,6 \\
Total & 81 & 41,7 & 113 & 58,2 & 194 & 100 \\
\hline
\end{tabular}

A prevalência de anemia encontrada na amostra foi de 41,7 \%, com predominância na classe socioeconômica C. Foi constatada a presença de anemia em $23,2 \%$ dos adolescentes no estágio de $10-13$ anos e de $18,5 \%$ no estágio de 14-18 anos, não apresentando diferenças significativas $(p=0,16276)$ entre ambos os grupos etários (Tabela 4). Embora a prevalência de anemia tenha sido elevada, o estado nutricional dos adolescentes não apresentou influência sobre este resultado $(\mathrm{p}=0,914593)$, considerando que a maioria dos adolescentes eram eutróficos.

Von Der Heyde (1999) avaliou o estado nutricional de atletas adolescentes, inclusive da região Norte, e detectou valores médios de hemoglobina de $14,55 \mathrm{~g}_{\mathrm{d}} \mathrm{dl}^{-1}$ e prevalência de $15 \%$ de anemia no gênero masculino. Lima (2002) em estudo realizado com 513 adolescentes de escolas públicas de cinco zonas urbanas da cidade de Manaus/AM constatou uma prevalência de $7 \%$ de anemia no gênero masculino com valores médios de hemoglobina de $13,5 \mathrm{~g}^{-\mathrm{dl}^{-1}} \mathrm{e} 14,6 \mathrm{~g} \cdot \mathrm{dl}^{-1}$ nos estágios de 10-13 e 14-18 anos, respectivamente.

A prevalência de anemia ferropriva $(41,8 \%)$ observada no presente estudo mostrou-se maior que os resultados encontrados na literatura. Tal achado é considerado preocupante, uma vez que a anemia está associada à fadiga e ao baixo rendimento escolar e esportivo.

A alimentação adequada é o principal instrumento no combate a deficiência de ferro e anemia, sobretudo nos países em desenvolvimento, onde ocorre um baixo consumo de alimentos como carnes, frutas e hortaliças (Demaeyer, 1989).

Tabela 3 - Concentração de hemoglobina (média, mediana, mínimo e máximo) de atletas adolescentes da Fundação Vila Olímpica de Manaus-AM, 2003.

\begin{tabular}{ccccc}
\hline \multirow{2}{*}{ Estágio de vida } & \multicolumn{3}{c}{ Concentração de hemoglobina $\left(\mathrm{g} \mathrm{dl}^{-1}\right)$} & \\
& Média (DP) & Mediana & Mínimo & Máximo \\
& & & & \\
\hline 10-13 Anos & $12,5(1,4)$ & 12,5 & 9,9 & 16,2 \\
14-18 Anos & $13,3(1,4)$ & 13,1 & 10,0 & 17,3 \\
\hline
\end{tabular}

DP - Desvio padrão.

Tabela 4 - Distribuição de atletas adolescentes anêmicos e não-anêmicos segundo o estágio de vida, da Fundação Vila Olímpica de Manaus-AM, 2003.

\begin{tabular}{lcccccc}
\hline $\begin{array}{l}\text { Estágio } \\
\text { de vida }\end{array}$ & $\mathrm{n}$ & $\begin{array}{c}\text { Anêmicos } \\
\%\end{array}$ & $\mathrm{n}$ & $\begin{array}{c}\text { Não anêmicos } \\
\%\end{array}$ & $\mathrm{n}$ & \multicolumn{1}{c}{$\begin{array}{c}\text { Total } \\
\%\end{array}$} \\
\hline $\begin{array}{l}10-13 \\
\text { Anos }\end{array}$ & 45 & 23,2 & 63 & 32,5 & 108 & 55,7 \\
$\begin{array}{l}14-18 \\
\text { Anos }\end{array}$ & 36 & 18,5 & 50 & 25,8 & 86 & 44,3 \\
Total & 81 & 41,7 & 113 & 58,3 & 194 & 100 \\
\hline
\end{tabular}


Os adolescentes deveriam receber educação nutricional e condiçôes que os habilitassem a manter uma dieta equilibrada, evitando desse modo eventuais doenças por deficiências de nutrientes (Eisenstein, 2000).

\section{CONCLUSÃO}

A alta prevalência de anemia evidenciada no presente estudo denota um desequilíbrio nutricional neste grupo populacional, fator que pode vir a predispor o aparecimento e o desenvolvimento de disfunções orgânicas e interferir negativamente no crescimento físico e rendimento esportivo dos atletas adolescentes analisados.

A adoção de medidas preventivas de educação nutricional com a difusão de conceitos quanto a uma alimentação saudável aliada à atividade física, na tentativa de contribuir para a melhoria das condiçōes de saúde e do rendimento esportivo desses atletas adolescentes seria uma importante medida a ser tomada. Diante do exposto, sugere-se a implementação de medidas efetivas de intervenção e educação nutricional visando a minimização e/ou o controle da anemia ferropriva.

\section{AGRADECIMENTOS}

À FINEP/PPG7, Proc. 64.99.0477.00, pelo suporte financeiro.

\section{BIBLIOGRAFIA CITADA}

Camargo, M.A.C.; Santos, I.D.C. 1997. Perfil socioeconômico e cultural do estudante de graduação da Universidade Federal do Paraná, Curitiba. 86 pp.

DeMaeyer E.M. 1989. Preventing and controlling iron deficiency anaemia through primary health care. World Health Organization. Geneva. 58pp.

Dolye, E.I; Feldman, R.H.L. 1997. Factors affeting nutrition behavior among middle-class adolescents in urban area of Northern region of Brazil. Rev. Saúde Pública, 31: 342-50.

Eisenstein, E.; Coelho, K.S.C.; Coelho, S.C.; Coelho, M.A.S.C. 2000. Nutrição na adolescência. J. Pediatria, 76(3): S263S274.

Fundo Das Naçôes Unidas Para a Infância (UNICEF); United Nations University (UNU); World Health Organization (WHO); Micronutrient Initiative (MI). 1998. Preventing Iron Deficiency in Women and Children: technical consensus on key issues and resources for programme advocacy, planning and implementation. UNICEF, New York. p. 7-9.
Heyward V.H.; Stolarczyk, L.M. 2000. Método antropométrico. In: Heyward V.H. Avaliação da composiçāo corporal aplicada. Tradução de Sérgio H. F. Carvalho. São Paulo: Manole, p. 73-98.

Lima, M. 2002. Anemia ferropriva em adolescentes escolares de Manaus - $A M$. Dissertação de Mestrado, Universidade Federal do Amazonas, Manaus, Amazonas. 89pp.

McArdle, W.D.; Katch, F.I.; Katch, V.L. 1998. Fisiologia do exercício: energia, nutrição e desempenho humano. 4. ed. Rio de Janeiro: Guanabara Koogan.

MS - Ministério da Saúde. 2004. Compromisso social para a redução da anemia por carência de ferro no Brasil. 9p. (http://dtr2004. saude.gov.br/nutricao/ferro.php). Acesso: 23/03/2008.

MS - Ministério da Saúde, UNICEF. 2007. Cadernos de Alimentação básica: Carências de Micronutrientes. (Série A. Normas e Manuais Técnicos). 60pp.

Nascimento, D.; Siebert, A.N.; Grillo, L.P.; Godry, R.C. 2004. Características antropométricas e bioquímicas de atletas infantojuvenis pertencentes ao projeto social "Criança feliz no tênis, feliz na escola” Revista Nutrição em Pauta, 67: 44-49.

National Center For Health Statistics. 2000. Centers of disease and control. 89pp. Disponível em http://www.cdc.gov/growthcharts. Acesso em 05/11/2003.

Oliveira, J.S.R.; Figueiredo, M.S. 1998. Anemias R B M, 55: 191213.

Silva, J.J. 1992. Características antropométricas e de aptidão física em escolares amazonenses. Dissertação de Mestrado, Universidade de São Paulo, São Paulo. 117pp.

Thompson, J. L. 1998. Energy balance in young athletes. J. Sports Nutrition, 8:160-174.

Tsuyuoka R.; Bailey J.W.; Nery Guimarães, A.M.; Gurgel, R.Q.; Cuevas, L.E. 1999. Anemia and intestinal parasitic infections in primary school students in Aracaju, Se, Brazil. Cad Saude Publica, 15(2):413-21.

Von Der Heyde, M.E.D. 1999. Avaliação da composição corporal, nivel de hemoglobina e perfil nutricional de atletas adolescentes. Tese de Doutorado, Universidade Federal de São Paulo. São Paulo. 178pp.

World Health Organization. 1995. Physical Status: the use and interpretation of anthropometry. Geneva: WHO. Who Technical Report Series, 854.

Recebido em 15/10/2004

Aceito em 07/04/2008 